\title{
Near-field visualization of electromagnetically induced transparency in resonant terahertz structures
}

\author{
A. Halpin* and A. Bhattacharya* and C. Mennes ${ }^{\dagger}$ and J. Gómez-Rivas*ł \\ ${ }^{*}$ Dutch Institute for Fundamental Energy Research (DIFFER), Eindhoven, Netherlands \\ ${ }^{\dagger}$ Dutch Institute for Atomic, Molecular and Optical Physics (AMOLF), Amsterdam, Netherlands. \\ ‡COBRA Research Institute, Eindhoven University of Technology, Eindhoven, Netherlands. \\ Email: a.halpin@differ.nl
}

\begin{abstract}
We present a study on a dolmen structure, where a bright resonator couples to a dark resonator, to investigate nearfield signatures of electromagnetically induced transparency. We demonstrate an enhancement of the near-fields in the spectral vicinity of the transparency, and observe mode hybridization resulting from the coupling between the two resonant structures.
\end{abstract}

\section{INTRODUCTION AND BACKGROUND}

$\mathbf{R}$ ESONANT structures and metamaterials offer an attractive canvas for the design of novel optoelectronic devices in the far-infrared. By exploiting the strong scattering properties of metallic resonators, effects such as beam steering [1], spectral filtering [2], and enhanced group delays [3] have been demonstrated over thin optical path lengths with high figures of merit.

In many implementations, such effects arise due to interferences occurring in local fields of the resonators via evanescent coupling. We use scanning probe near-field $\mathrm{THz}$ time-domain microscopy to present spectral maps of an array of gold dolmens, consisting of one resonator oriented along the incident field polarization and another oriented orthogonally. This geometry was first introduced at optical frequencies, demonstrating plasmon-induced transparency [4] and mode hybridization [5] measured using NSOM. At those frequencies only intensity enhancements in the near field were measured, however at $\mathrm{THz}$ frequencies the near-field distributions can be characterized in both amplitude and phase.

The system consists of a first resonator that supports a bright mode, which couples efficiently to the radiation field, and a second resonator that supports a dark mode which cannot be driven by incident plane wave radiation. The interaction of these two modes in the near-field gives rise to electromagnetically induced transparency observed in far-field extinction spectra, where the frequencies transmitted by the structure experience a large group delay.

\section{RESUlts}

The geometry of the sample is shown in the inset to Fig. 1 . The gold dolmens consist of a top horizontal rod of $150 \mu \mathrm{m}$ length and $40 \mu \mathrm{m}$ in width, and two vertical rods of 140 $\mu \mathrm{m}$ and $40 \mu \mathrm{m}$ in width. The structures are arranged in a periodic array on a quartz substrate using photolithography, where the pitches of the array are $p_{x}=250 \mu \mathrm{m}$ and $p_{y}=290$ $\mu \mathrm{m}$. We employ a THz-TDS spectrometer (Menlo Systems $\mathrm{GmbH})$ to measure far-field extinction spectra, and a series of semiconductor $\mathrm{THz}$ microprobes consisting of miniaturized photoconductive switches [6] (Protemics GmbH, Germany) to measure near-fields for each Cartesian component, separately.

The latter results are then be used to generate spatial maps of electric field amplitude and phase as a function of frequency. In Fig. 1 we show the measured extinction spectrum of the dolmen sample in solid black, with a rescaled extinction spectrum for a random array of the top resonators overlaid in grey, highlighting the transparency supported by the combined system at $\sim 0.55 \mathrm{THz}$.

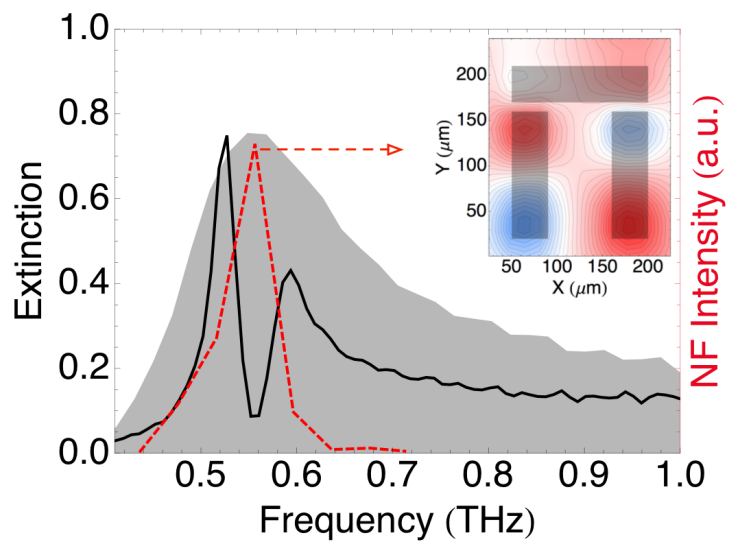

Fig. 1. Extinction spectra of the dolmen sample (solid black) and the uncoupled bright resonator (grey overlay). The near field spectrum at the bottom of the leftmost vertical rod is shown in dashed red. Inset: near-field map for the real part of the out of plane component of the electric field corresponding to the frequency of the transparency observed in the far-field.

For comparison, in dashed red we also plot the intensity spectrum of the out-of-plane field component recorded at the bottom of one of the dimer rods, where we observe a maximal enhancement at the frequency of the far-field transparency. This near-field enhancement arises from a quadrupolar mode in the vertical rod pair indirectly excited via the polarization induced in the top rod by the incident $\mathrm{THz}$ pulse, as seen in the inset to Fig. 1. Moreover, at frequencies outside the transparency window, the interaction between the two constituent resonators of the dolmen gives rise to the hybridization of the dipolar- and quadrupolar-like resonances supported by the horizontal and vertical rods, respectively. 
These two hybrid modes are consistent with the peaks observed in the extinction spectrum of Fig. 1, where electrostatic repulsion and attraction explain the shifts in their frequencies from the bare modes. At $0.56 \mathrm{THz}$ where the transparency window is observed in the far-field, we observe that the electric field is strongly enhanced in the vicinity of the dark resonator, and that the dipolar response of the top rod is almost fully suppressed. The absence of spectral shifts between near and far field features is notable and likely due to the relatively low losses in the system [7].

\section{CONCLUSION}

$\mathrm{THz}$ near-field microscopy was used to study coupling between bright and dark modes supported by a dolmen in a periodic array. The far-field extinction spectrum of the sample exhibits electromagnetically induced transparency, and the near-field measurements retrieve the mode hybridization and field enhancement about the dark resonator which gives rise to the far-field response.

\section{ACKNOWLEDGEMENT}

We acknowledge the financial support of the ERC through grant no.: 259272 THZ-PLASMON (2010) and 665619 MicroMap (2014). We also thank financial support of the Foundation for Fundamental Research on Matter (FOM), and Netherlands Organization for Scientific Research (NWO).

\section{REFERENCES}

[1] Tom P. Steinbusch, Hemant K. Tyagi, Martijn C. Schaafsma, Giorgos Georgiou, and Jaime Gómez Rivas, "Active terahertz beam steering by photo-generated graded index gratings in thin semiconductor films", Opt. Express 22, 26559, 2014.

[2] Xianliang Liu, Tatiana Starr, Anthony F. Starr, and Willie J. Padilla, "Infrared spatial and frequency selective metamaterial with near-unity absorbance", Phys. Rev. Lett. 104, 207403, 2010.

[3] Fumiaki Miyamaru, Hiroki Morita, Yohei Nishiyama, Tsubasa Nishida, Toshihiro Nakanishi, Masao Kitano and Mitsuo W. Takeda, "Ultrafast optical control of group delay of narrow-band terahertz waves", Sci. Rep. 4, 4346, 2014.

[4] Shuang Zhang, Dentcho A. Genov, Yuan Wang, Ming Liu, and Xiang Zhang, "Plasmon-induced transparency in metamaterials", Phys. Rev. Lett. 101, 047401, 2008.

[5] Ziliang Ye, Shuang Zhang, Yuan Wang, Yong-Shik Park, Thomas Zentgraf, Guy Bartal, Xiaobo Yin, and Xiang Zhang. "Mapping the near-field dynamics in plasmon-induced transparency", Phys. Rev. B 86, 155148, 2012.

[6] Markus Wächter, Michael Nagel, and Heinrich Kurz, "Tapered photoconductive terahertz field probe tip with subwavelength spatial resolution" Appl. Phys. Lett., 95, 041112, 2009.

[7] Arkabrata Bhattacharya, Giorgos Georgiou, Simon Sawallich, Christopher Matheisen, Michael Nagel, and Jaime Gómez Rivas, "Large near-to-far field spectral shifts for terahertz resonances", Phys. Rev. B 93, 035438, 2016. 